\title{
Malaria grave en unidad de cuidados intensivos: Reporte de un caso de una especie no endémica en Norte de Santander, Colombia
}

\author{
Severe Malaria in the Intensive Care Unit: The report of a Case of a \\ Non-Endemic Species in North of Santander, Colombia
}

\section{Malária grave na unidade de terapia intensiva: Informe de um caso de um tipo não endêmica no estado de Norte de Santander, na Colômbia}

\author{
Guillermo Ortiz-Ruiz, MD., Esp., PhD. * \\ Zulma Esperanza Urbina-Contreras, MD., Esp. ** \\ Andrés Felipe Lamos-Duarte, MD., Esp. *** \\ María Fernanda Ferreira, MD. **** \\ Francisco García-Zambrano, MD. *****
}

\begin{abstract}
Introducción: La malaria es una enfermedad infecciosa vectorial de predominio en regiones tropicales y subtropicales. Existen 5 serotipos de Plasmodium, en Colombia se encuentran dos serotipos para malaria endémica, $P$. vivax y $P$. falciparum. Norte de Santander es una zona endémica para $P$. vivax. Objetivo: Presentación de un caso de malaria grave importada por $P$. falciparum, con una breve descripción de los aspectos fisiopatológicos de la malaria grave y los advenimientos de las nuevas terapias antipalúdicas. Presentación del caso: Paciente masculino
\end{abstract}

de 45 años procedente de una región endémica para $P$. falciparum, que ingresó por cuadro febril inespecífico, trombocitopenia severa, alteración de la función renal y hepática con deterioro de su estado general. Es trasladado a la unidad de cuidados intensivos como urgencia dialítica, se diagnóstica malaria grave por $P$. falciparum, es tratado con antimaláricos y se reporta posible coinfección para el virus del dengue por inmunoglobulina $\mathrm{M}$ positiva (IgM), recibe terapia de reemplazo renal. Se contextualiza bajo un círculo vicioso en la disfunción de órganos, estructurado entre la insuficiencia renal y la insuficiencia respiratoria aguda con incremento de la permeabilidad vascular e hipoxemia refractaria, pese al esfuerzo terapéutico fallece por falla

Médico, especialista en Medicina Interna, especialista en Medicina Crítica y Cuidado Intensivo, especialista en Epidemiología, doctorado en Medicina Traslacional, coordinador Clínica Santa Clara, Bogotá, Cundinamarca, Colombia.

** Médica, especialista en Medicina Interna, especialista en Medicina Crítica y Cuidado Intensivo, Hospital Universitario Erasmo Meoz, Cúcuta, Norte de Santander, Colombia.

*** Médico, especialista en Epidemiología, docente Universidad de Boyacá, Tunja, Boyacá, Colombia. Médico, Unidad de Cuidado Intensivo Adultos, Hospital Universitario Erasmo Meoz, Cúcuta, Norte de Santander, Colombia

**** Médica, Unidad de Cuidados Intensivos, Hospital Universitario Erasmo Meoz, Cúcuta, Norte de Santander, Colombia.

*****Médico, Hospital San Juan de Dios de Arboleda, Arboledas, Norte de Santander, Colombia.

Correspondencia: Andrés Felipe Lamos Duarte. Calle 0 No. 4E-74, Barrio Quinta Bosch, Cúcuta, Norte de Santander, Colombia. Teléfono: +57 3125707455. Email: cptpipen@gmail.com 
orgánica múltiple, por malaria grave. Conclusiones: La malaria es un problema en el área de salud pública, en nuestro caso corresponde a una malaria importada ya que en el departamento de Norte de Santander no se ha identificado dicho serotipo. [Ortiz-Ruiz G, Urbina-Contreras ZE, Lamos-Duarte AF, Ferreira MF, García-Zambrano F. Malaria grave en unidad de cuidados intensivos: Reporte de un caso de una especie no endémica en Norte de Santander, Colombia. MedUNAB 2017-2018; 20(3): 383-392].

Palabras clave: Malaria; Malaria Falciparum; Síndrome de Dificultad Respiratoria del Adulto; Insuficiencia Renal; Unidad de Cuidados Intensivos; Cuidados Críticos.

\section{Resumen}

Introduction: Malaria is a vector-borne infectious disease which is predominant in tropical and subtropical regions. There are 5 serotypes of Plasmodium, in Colombia there are two serotypes for endemic malaria, P. vivax and P. falciparum. North of Santander is an endemic area for $P$ vivax. Objective: To show a case of severe malaria caused by $P$. falciparum, with a brief description of the pathophysiological aspects of severe malaria and the advent of new antimalarial therapies. Case Presentation: A 45year-old male patient from an endemic region with $P$. falciparum, who was admitted due to nonspecific febrile symptoms, severe thrombocytopenia, impaired renal and hepatic functions with deterioration of his general condition. $\mathrm{He}$ is transferred to the intensive care unit as a dialytic urgency. Severe malaria due to P. falciparum was diagnosed, he is treated with antimalarial medication, and a possible coinfection is reported for the dengue virus due to a positive immunoglobulin $\mathrm{M}$ (IgM) result, so he receives a renal replacement therapy. The case is contextualized in a vicious circle of organ dysfunction, which is structured between renal failure and acute respiratory failure with an increased vascular permeability and refractory hypoxemia; despite the therapeutic effort, the patient dies due to multiple organ failures, and severe malaria. Conclusions: Malaria is a problem in the public health area. This case corresponds to imported malaria because this serotype has not been identified yet in the department of North of Santander. [OrtizRuiz G, Urbina-Contreras ZE, Lamos-Duarte AF, Ferreira MF, Garcia-Zambrano F. Severe Malaria in the Intensive Care Unit: The report of a Case of a Non-Endemic Species in

\section{Introduction}

Desde los cimientos de la medicina clásica, algunos médicos como Hipócrates; pensaron que era atribuida a los miasmas o "aires malos" de las lagunas y pantanos de donde provienen sus nombres, malaria (mal aire) y paludismo (plauster o pantano) (1). La malaria es una enfermedad infecciosa transmitida por el mosquito hembra del género Anopheles. Existen cinco especies de Plasmodium de interés médico (2), con un espectro endémico en 91 países, que ponen en riesgo al $40 \%$ de la población mundial (3) (Figura 1).
North of Santander, Colombia. MedUNAB 2017-2018; 20(3): 383-392].

Keywords: Malaria; Malaria, Falciparum; Respiratory Distress Syndrome, Adult; Renal Insufficiency; Intensive Care Units; Critical Care.

\section{Resumo}

Introdução: A malária é uma doença infecciosa vetorial predominantemente nas regiões tropicais e subtropicais. Existem 5 sorotipos de Plasmodium, na Colômbia existem dois sorotipos para malária endêmica, $P$. vivax e $P$. falciparum. O estado de Norte de Santander é uma região endêmica para P. vivax. Objetivo: Apresentação de um caso de malária grave importado por $\mathrm{P}$. falciparum, com uma breve descrição dos aspectos fisiopatológicos da malária grave e o advento das novas terapias anti-maláricas. Apresentação do caso: Paciente do sexo masculino de 45 anos de uma região endêmica do P. falciparum, admitido com sintomas febris não específicados, trombocitopenia grave, insuficiência renal e função hepática, num estado geral deteriorado. Ele é transferido para a unidade de cuidados intensivos como emergência de diálise, é diagnosticada a malaria grave causada pelo P. falciparum, ele é tratado com antimaláricos e se reporta uma posível co-infecção pelo vírus da dengue pela imunoglobulina $\mathrm{M}$ (IgM) positiva, recebe então, tratamento de reposição renal. É contextualizado num círculo vicioso na disfunção dos órgãos, estruturado entre insuficiência renal e insuficiência respiratória aguda com aumento da permeabilidade vascular e hipoxemia refractária, apesar do esforço terapêutico morre devido à falência múltipla de órgãos, gerada pela malária grave. Conclusões: A malária é um problema na área da saúde pública, no nosso caso corresponde a uma malária importada, já que no departamento de Norte de Santander este serótipo não foi identificado. [Ortiz-Ruiz G, Urbina-Contreras ZE, LamosDuarte AF, Ferreira MF, García-Zambrano F. Malária grave na unidade de terapia intensiva: Informe de um caso de um tipo não endêmica no estado de Norte de Santander, na Colômbia. MedUNAB 2017-2018; 20(3): 383-392].

Palavras-chave: Malária; Malária Falciparum; Síndrome do Desconforto Respiratório do Adulto; Insuficiência Renal; Unidades de Terapia Intensiva; Cuidados Críticos.

Se estima un total de 207 millones de casos en el año 2012, de los cuales 627,000 fueron muertes en menores (4-10). En Colombia, el número de muertes que registró el Departamento Administrativo Nacional de Estadísticas (DANE) durante el periodo de 1999-2009 fue entre 65 y 165 muertes al año y se registraron 150,000 casos anuales por Plasmodium falciparum (5) (Figura 2).

De acuerdo con los datos epidemiológicos del Sistema de Salud Pública (SIVIGILA) en el departamento de Norte de Santander se estimó una tasa de incidencia para el 2010 de 335 casos por 100,000 habitantes para malaria (11-19). En 


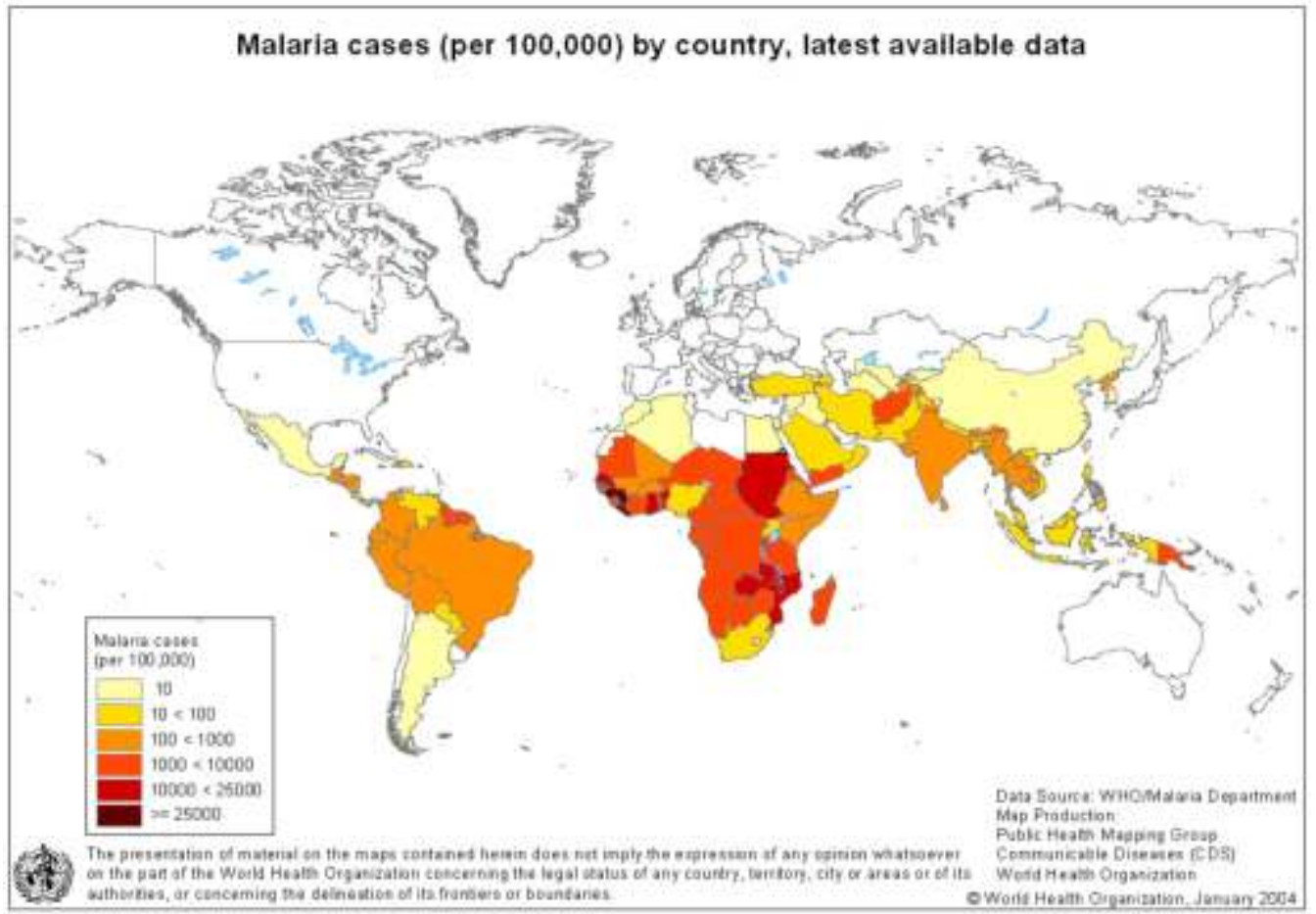

Fuente: Tomado de: Organización Panamericana de la Salud, Oficina Regional de la Organización Mundial de la Salud. Esquemas de tratamiento: México, América Central, Hispaniola. Disponible en www.paho.org/spanish/ad/dpc/cd/mal-2005-cor-12.ppt\#1

Figura 1. Distribución mundial de la malaria.

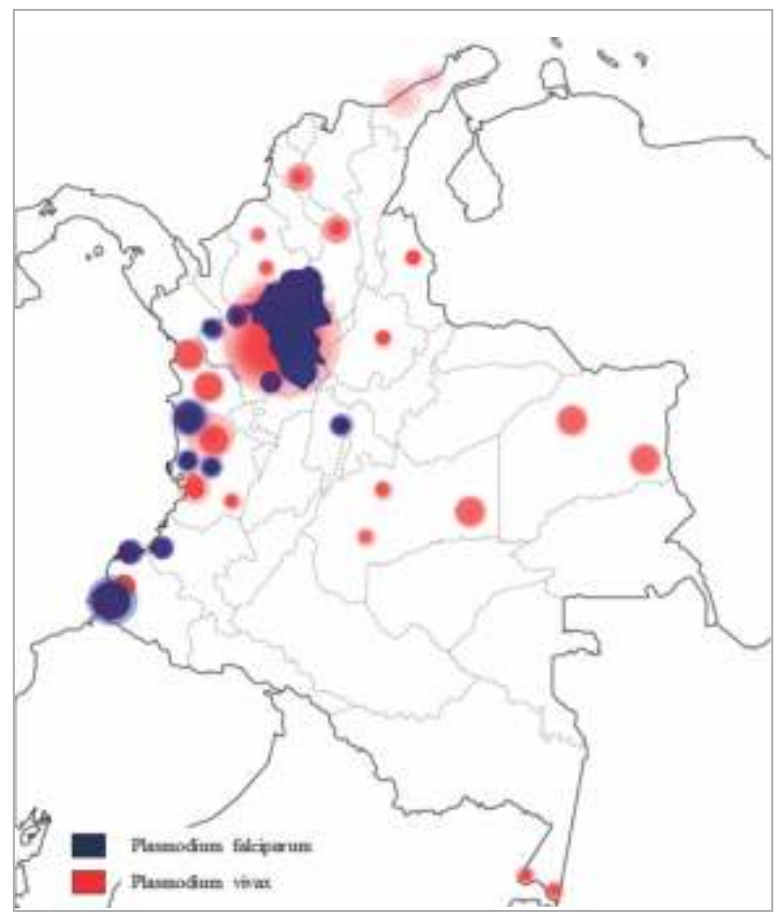

Fuente: Elaboración de los autores.

Figura 2. Distribución del Plasmodium según áreas endémicas en Colombia 
nuestro país se adelanta, desde el año 2010, el plan para malaria que busca desarrollar estrategias para la prevención, tratamiento oportuno y disminución de la mortalidad en áreas endémicas $(6,20)$.

Se presenta un caso de malaria grave por Plasmodium falciparum importada de un área endémica a la ciudad de Cúcuta, paciente que ingresó con criterios de severidad, requirió terapia multisoporte en Unidad de Cuidados Intensivos (UCI), pese al esfuerzo terapéutico dirigido fallece en el contexto de falla multiorgánica. El objetivo es plantear una breve descripción de los aspectos fisiopatológicos de la malaria grave y los advenimientos de las nuevas terapias antipalúdicas contrastando con la terapéutica aplicada al caso en mención.

\section{Reporte del caso}

Paciente masculino de 45 años, natural de Bogotá, con antecedente de alcoholismo activo desde hace 3 años; se desempeñaba como transportador con nexo a zonas aledañas al municipio de Buenaventura. Inicialmente, cursa con cuadro febril, hiporexia, dolor abdominal, vómitos y síntomas urinarios inespecíficos; con reporte de paraclínicos: leucocitosis, neutrofilia, trombocitopenia y uroanálisis sugestivo de Infección de Vías Urinarias (IVU), que ameritó manejo intrahospitalario por 3 días; persiste sintomático y es remitido a nuestra institución sin diagnóstico.

Ingresó al servicio de urgencias, por clínica de 13 días de episodios febriles intermitentes, escalofríos, y signos ocasionales de intolerancia a la vía oral de aspecto bilioso, tendencia a las deposiciones líquidas, dolor abdominal moderado en hemiabdomen derecho con sintomatología urinaria y 3 días previos a la hospitalización presentó coluria. Signos vitales: Tensión Arterial (TA): 135/72 mmHg, Frecuencia Cardiaca (FC): 112 lpm, Frecuencia Respiratoria (FR): $24 \mathrm{rpm}$, Temperatura (T): $38.2{ }^{\circ} \mathrm{C}$, Saturación de Oxigeno (SO2): 90\%; ictericia generalizada, hepatomegalia dolorosa y edema bimaleolar. Paraclínicos de ingreso: trombocitopenia marcada, transaminasas elevadas, deshidrogenasa láctica marcada, disociación Nitrógeno Ureico en Sangre (BUN)/creatinina 3:1, hiperbilirrubinemia severa conjugada, acidosis metabólica moderada con disfunción pulmonar. Ecografía abdominal que informa colecistitis acalculosa, el servicio de cirugía general valoró el escenario y descarto abdomen agudo quirúrgico. En este contexto requirió transfusión de 8 unidades de plaquetas, se realiza diferencial con salmonelosis y enfermedad por vectores. Se descartó IVU por urocultivo negativo.

Considerando enfermedad tropical se solicita test para leptospira y dengue; sin embargo, por el deterioro clínico progresivo y la acidosis metabólica severa persistente se traslada a UCI. El primer día en UCI se solicitó gota gruesa con reporte de hiperparasitemia por Plasmodium falciparum, se inició terapia antipalúdica según las guías del Ministerio Colombiano en el contexto de malaria con criterios de severidad, con artemether/lumefantrine por tres días, clindamicina y quinina por 7 días. Así mismo, evoluciona desfavorablemente con trombocitopenia severa, se trasfunde plaquetas y se documentó gasometría con pH: 7.28, $\mathrm{HCO} 3$ (Bicarbonato): $15 \mathrm{mEq} / 1, \mathrm{PaCO} 2$ (Presión parcial de dióxido de carbono en la sangre arterial): $38 \mathrm{~mm} / \mathrm{Hg}$ y $\mathrm{PaO} 2 / \mathrm{FiO} 2$ (Relación entre la tensión arterial de oxígeno y la fracción inspirada de oxigeno): 240 , FiO2 (Fracción inspirada de oxigeno): 50\%; además, se informó antígeno de superficie contra el virus de la hepatitis B (Ags-VHB) y anticuerpos para el virus A y C (Anti-VHA, Anti-VHC) negativos, antígenos febriles igualmente negativos, IgM para dengue positivo y albúmina: $2.1 \mathrm{~g} / \mathrm{dl}$. Refirió cefalea holocraneana con Tomografía Axial Computarizada (TAC) de cráneo simple sin alteraciones y TAC de tórax simple con derrame pleural bilateral no significativo. Desde el punto de vista renal, creatinina: 5.4 $\mathrm{mg} / \mathrm{dl}$, BUN: $110 \mathrm{mg} / \mathrm{dl}$ en rango de oligoanuria, por lo cual se indicó de terapia de soporte renal, APACHE II: 11 puntos (Acute Physiology And Chronic Health Evaluation 11), SOFA: 10 puntos (Sequential Organ Failure Assessment Score).

El segundo día, paciente con diagnósticos de malaria severa, trombocitopenia severa secundaria, falla renal aguda AKIN III (Acute Kidney Injury Network) con criterios de urgencia dialítica y disfunción orgánica SOFA 12 puntos y APACHE II de 28 puntos, por lo que se insistió en la Terapia de Soporte Renal (TSR). En su tercer día, persistencia de falla renal aguda con creatinina: $6.2 \mathrm{mg} / \mathrm{dl}, \mathrm{BUN}: 128 \mathrm{mg} / \mathrm{dl}$, gasometría $\mathrm{pH}$ : 7.21, $\mathrm{HCO} 3: 14 \mathrm{mEq} / 1, \mathrm{CO} 2: 37 \mathrm{~mm} / \mathrm{Hg}$, $\mathrm{PaO} 2 / \mathrm{fIO} 2:$ 196. Al cuarto día, presenta deterioro respiratorio, se practica radiografía de tórax con congestión pulmonar bilateral y demás paraclínicos estacionarios. Evolución clínica tórpida que requirió ventilación mecánica invasiva en modo asistencial controlado, soporte vasopresor e inicio de terapia dialítica diaria. El sexto día en UCI, evoluciona con signos de respuesta sistémica por sospecha de foco pulmonar, Escala de Valoración Clínica de Infección Pulmonar (CPIS) de 6 puntos, se inicia terapia antimicrobiana empírica de amplio espectro.

El séptimo día en UCI, tolera terapia de sostén renal, con parámetros ventilatorios y soporte vasopresor elevados. Al octavo día en UCI, paciente en falla multiorgánica en contexto de Shock séptico con escala de falla orgánica (SOFA) de 15 puntos y APACHE II 28 puntos, índice de oxigenación de 20, disfunción pulmonar severa, con indicación de referencia a centro médico con disponibilidad de Oxigenación con Membrana Extracorpórea (ECMO). Se documentó paraclínicos de control con índice parasitario no detectable. Se aísla de secreción bronquial Acinetobacter baumannii multirresistente sensible a tigeciclina - colistin y se ajusta terapia antimicrobiana dirigida. 
El noveno día en UCI, paraclínicos de control con creatinina $3.4 \mathrm{mg} / \mathrm{dl}$, BUN $75 \mathrm{mg} / \mathrm{dl}$, con Síndrome de Respuesta Inflamatoria Sistémica (SIRS) activo y con conteo plaquetario en ascenso $115,000 / \mathrm{mm}^{3}$, pero sin mejoría en las otras fallas orgánicas con SOFA:18 puntos, APACHE II: 34 puntos y lactato: $6 \mathrm{mmol} / \mathrm{L}$; bilirrubina total: $8 \mathrm{mg} / \mathrm{dl}$ y bilirrubina directa: $5 \mathrm{mg} / \mathrm{dl}$, transaminasas TGO: 158U/L, TGP: 170U/L, gasimetría pH:7.21, HCO3: $14 \mathrm{mEq} / \mathrm{l}, \mathrm{CO} 2: 37 \mathrm{~mm} / \mathrm{Hg}, \mathrm{PaO} 2 / \mathrm{fIO} 2: 120$ (Tabla 1), con modo Ventilatorio con Liberación de Presión (APRV) y Presión Positiva al Final de la Expiración (PEEP) 36 cmH2O. Se consideró escenario de sepsis pulmonar por germen multirresistente y Síndrome de Dificultad Respiratoria Aguda (SDRA) del adulto con indicación de terapia ECMO. Durante el día 13 en UCI, el paciente fallece por parada cardiaca en asistolia.

\section{Discusión}

Se trató de un caso de malaria severa, acorde con los criterios de severidad de la declaración de la Organización
Mundial de la Salud (OMS) para el año 2010 para este tipo de escenarios (21) (Tabla 2).

A Nivel Renal, la disrupción endotelial por expresión de receptores y citocinas, secuestro de glóbulos rojos, obstrucción vascular y vasoconstricción; estos eventos llevan a necrosis $(4,9)$. Histológicamente, la injuria renal aguda producida por $P$. falciparum es la necrosis tubular aguda (10).

En el curso del cuadro, presenta fracaso renal agudo que ameritó terapia de reemplazo renal, con pobre respuesta. De acuerdo a la literatura, la intervención temprana mejora los resultados renales y extrarrenales para pacientes con lesión renal aguda, esta se ha asociado una mortalidad del 15\% en los pacientes con diálisis por hemofiltración en lesión renal aguda por malaria por $P$. falciparum $(18,10)$.

Esto se contrasta con un reporte realizado en la India, en pacientes con lesión renal aguda por malaria $P$. falciparum, determinaron una mortalidad del $95 \%$ en pacientes con APACHE II mayor a 24 puntos y SOFA por encima de 12 puntos (22). Respecto a la acidosis metabólica, está dada por

Tabla 1. Control paraclínico.

\begin{tabular}{|c|c|c|c|c|c|c|}
\hline Extrahospitalarios & Ingreso & UCl día 1 & UCl día 2 & UCl día 3 & UCl día 8 & UCl día 9 \\
\hline $\begin{array}{c}\text { Cuadro hemático } \\
\text { Leucocitos: } \\
\text { 13,000/mm } \\
\text { Neutrófilos: } \\
65 \% \\
\text { Plaquetas: } \\
31,000 / \mathrm{mm}^{3} \\
\text { Uroanálisis } \\
\text { Nitritos:(+) } \\
\text { Leucocitos: } \\
15 \text { por campo } \\
\text { Bacterias:(++) }\end{array}$ & $\begin{array}{c}\text { Cuadro hemático } \\
\text { Leucocitos } 5,900 / \mathrm{mm}^{3} \\
\text { Neutrófilos:70\% } \\
\text { Hemoglobina } 16.5 \mathrm{~g} / \mathrm{dl} \\
\text { Hematocrito } 48 \% \\
\text { Plaquetas: } 2,000 / \mathrm{m}^{3} \\
\text { Perfil hepático } \\
\text { TGO: } 173 \mathrm{U} / \mathrm{dl} \\
\text { TGP: } 235 \mathrm{U} / \mathrm{dl} \\
\text { Bilirrubina total: } \\
9.2 \mathrm{mg} / \mathrm{dl} \\
\text { Bilirrubina directa: } \\
6.2 \mathrm{mg} / \mathrm{dl} \\
\text { LDH: } 1,300 \mathrm{U} / \mathrm{l} \\
\text { Perfil renal } \\
\text { BUN: } 70 \mathrm{mg} / \mathrm{dl} \\
\text { Creatinina: } 2.5 \mathrm{mg} / \mathrm{dl} \\
\text { Electrolitos séricos } \\
\text { Sodio: } 135 \mathrm{mEq} / \mathrm{l} \\
\text { Potasio: } 4.2 \mathrm{mEq} / \mathrm{l} \\
\text { Cloro: } 102 \mathrm{mEq} / \mathrm{l} \\
\text { Gasometría } \\
\text { PH: } 7.31 \\
\text { HCO3:16 mEq/l } \\
\text { PaCO2: } 36 \mathrm{~mm} / \mathrm{Hg} \\
\text { PaO2/FIO2: } 205 \\
\text { FIO2: } 40 \% \text {. }\end{array}$ & $\begin{array}{c}\text { Función renal: } \\
\text { Creatinina: } 5.4 \mathrm{mg} / \mathrm{dl} \\
\text { BUN: } 110 \mathrm{mg} / \mathrm{dl} \\
\text { Gota gruesa: } 285,000 \\
\text { parásitos/microlitro } \\
\text { Gasometría } \\
\text { PH: } 7.28 \\
\text { HCO3: } 15 \mathrm{mEq} / \mathrm{l} \\
\text { PaCO2: } 38 \mathrm{~mm} / \mathrm{Hg} \\
\text { PaO2/FiO2240 } \\
\text { FiO2:50\% } \\
\text { AgS-VHB yanticuerpos } \\
\text { para el virus AY C: } \\
\text { negativos } \\
\text { Antígenos febriles: } \\
\text { negativos } \\
\text { IgM para dengue: } \\
\text { positivo } \\
\text { Albúmina: } \\
2.1 \text { g/dl } \\
\text { APACHE II: } \\
11 \text { puntos } \\
\text { SOFA: } \\
10 \text { puntos }\end{array}$ & $\begin{array}{c}\text { SOFA:12 } \\
\text { puntos } \\
\text { APACHE } \\
\text { Il: } 28 \\
\text { puntos }\end{array}$ & $\begin{array}{c}\text { Función } \\
\text { renal: } \\
\text { Creatinina: } \\
6.2 \mathrm{mg} / \mathrm{dl} \\
\text { BUN: } \\
\text { 128mg/dl } \\
\text { Gasometría } \\
\text { PH: } 7,21 \\
\text { HCO3: } \\
\text { 14mEq/l } \\
\text { CO2: } \\
37 \mathrm{~mm} / \mathrm{Hg} \\
\mathrm{PaO} 2 / f 102: \\
196\end{array}$ & $\begin{array}{c}\text { SOFA:15 } \\
\text { puntos } \\
\text { APACHE II:32 } \\
\text { puntos } \\
\text { índice de } \\
\text { oxigenación } 20 \\
\text { PaO2/FiO2:160 }\end{array}$ & $\begin{array}{c}\text { Función } \\
\text { hepática } \\
\text { Bilirrubina total: } \\
8 \mathrm{mg} / \mathrm{dl} \\
\text { Bilirrubina } \\
\text { directa:5mg/dl } \\
\text { TGO: } 158 \mathrm{U} / \mathrm{L} \\
\text { TGP: } 170 \mathrm{U} / \mathrm{L} \\
\text { Perfil renal } \\
\text { Creatinina: } \\
3.4 \mathrm{mg} / \mathrm{dl} \\
\text { BUN: } 75 \mathrm{mg} / \mathrm{dl}, \\
\text { Plaquetas: } \\
\text { 115,000/mm } \\
\text { SOFA: } \\
\text { 18 puntos } \\
\text { APACHE II: } \\
34 \text { puntos } \\
\text { Lactato: } 6 \mathrm{mmol} / \mathrm{L} \\
\text { Gasometría } \\
\text { PH: } 721 \\
\text { HCO3: } 14 \mathrm{mEg} / \mathrm{l} \\
\text { CO2: } 37 \mathrm{~mm} / \mathrm{Hg} \\
\text { PaO2/flO2: } 120 \\
\text { con modo } \\
\text { ventilatorio APRV } \\
\text { PEEP } 36 \mathrm{~cm} \mathrm{H} 20\end{array}$ \\
\hline
\end{tabular}

Fuente: Elaboración de los autores. 
Tabla 2. Características de malaria severa

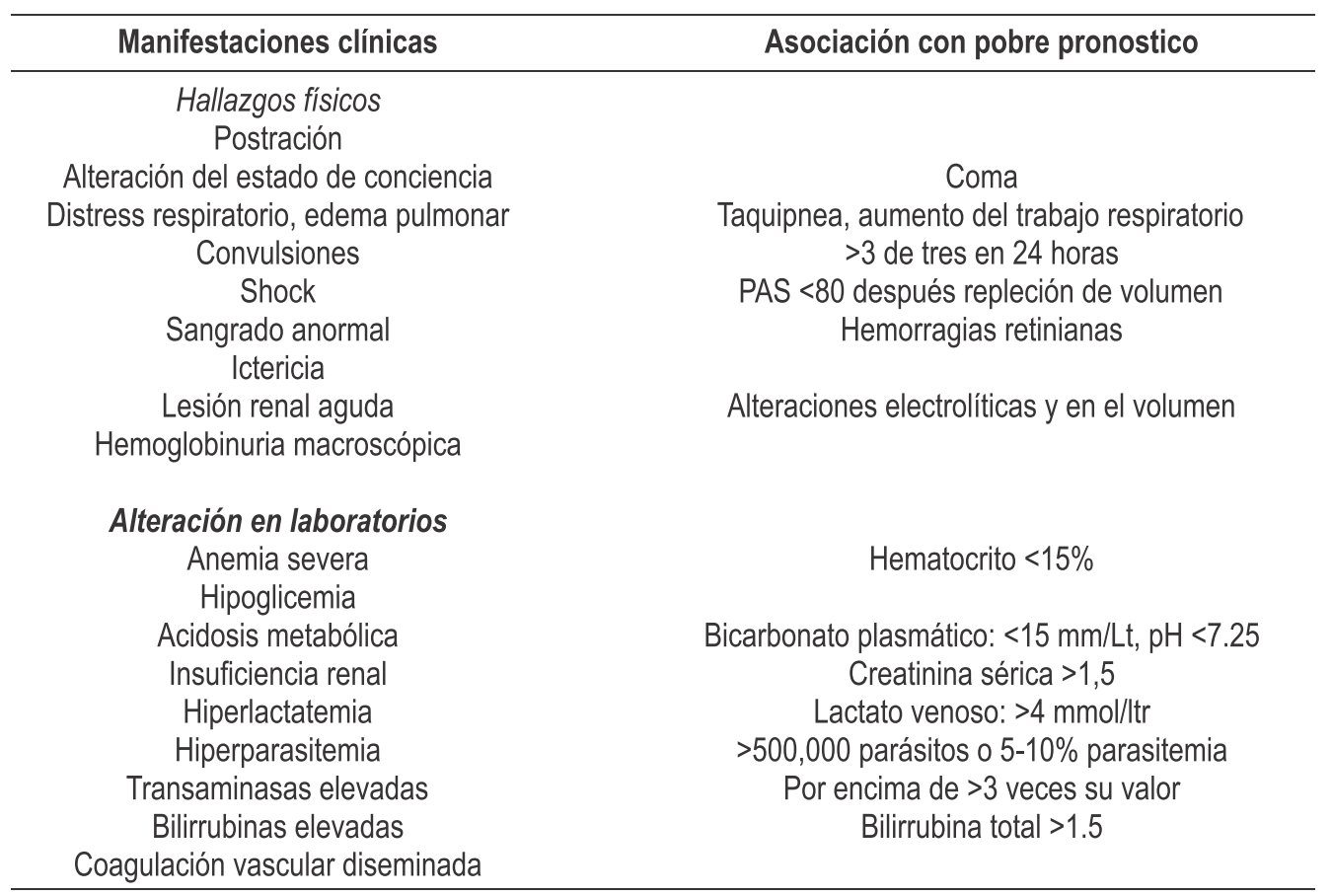

*PAS: Presión arterial sistólica.

Fuente: Elaboración de los autores.

la isquemia, hipovolemia, glucolisis anaerobia, en este caso se asoció una menor depuración de lactato causado por la hepatitis malárica y la alteración del balance hidroelectrolítico(4).

Los factores de mal pronóstico para la necesidad de diálisis en malaria grave con lesión renal aguda son: disfunción cerebral, hiperbilirrubinemia, SDRA, acidosis, Coagulación Intravascular Diseminada (CID), hemoglobinuria, anemia, oliguria o anuria a la admisión (4) (Figura 3).

A nivel respiratorio, la disrupción en la integridad de la membrana alveolo capilar, que conduce a una fuga del plasma hacia el intersticio alveolar, produciendo edema. El edema pulmonar en malaria con síndrome de dificultad respiratoria aguda (SDRA), no tiene origen cardiogénico (13). Como consecuencia, se forman membranas hialinas eosinofílicas cubriendo el alveolo con contenido proteináceo(Figura 4).

La anemia malárica grave se observa principalmente en las zonas endémicas; solo una serie de paludismo importado informó a los pacientes que presentaban anemia grave según la definición de la OMS (23). La trombocitopenia refractaria del paciente fue un marcador para mortalidad importante, esto se contrasta con la literatura donde existe una fuerte asociación con la duración prolongada de la hospitalización, el aumento de la necesidad de cuidados intensivos y el aumento de la mortalidad (24).
Estas complicaciones, se contrasta con lo reportado por Sahu, donde la incidencia de ictericia e insuficiencia renal en esta serie fue mayor (25). Tres criterios principales de la OMS que estuvieron presentes en las 24 horas posteriores a la admisión estuvieron fuertemente correlacionados con la mortalidad en la UCI: edema pulmonar, shock y acidosis metabólica.

Con respecto al diagnóstico, la gota gruesa es el método más difundido para la malaria y el recomendado como primera opción en el proceso de detección, con una sensibilidad de $61.6 \%$ y especificidad $95.1 \%$ cuando se compara con la Proteína C Reactiva(PCR) como referencia estándar(26).

En el contexto nacional, el diagnóstico diferencial de las demás patologías febriles hemorrágicas como el dengue, son una opción a tener en cuenta. En nuestro, caso el paciente presentaba una IgM para dengue positiva, la cual no especifica si el paciente estaba cursando con un episodio agudo a subagudo. La literatura describe positiva la IgM para dengue desde 5 días de aparición de los síntomas hasta 3 meses (27).

La primera línea de manejo para la malaria complicada por Plasmodium falciparum, es el artesunato endovenoso combinado (5). Las dosis deben administrarse por vía parenteral lo antes posible en todos los pacientes con malaria grave (Tabla 3). Existen dos clases de agentes parenterales para el tratamiento de la malaria severa: los 


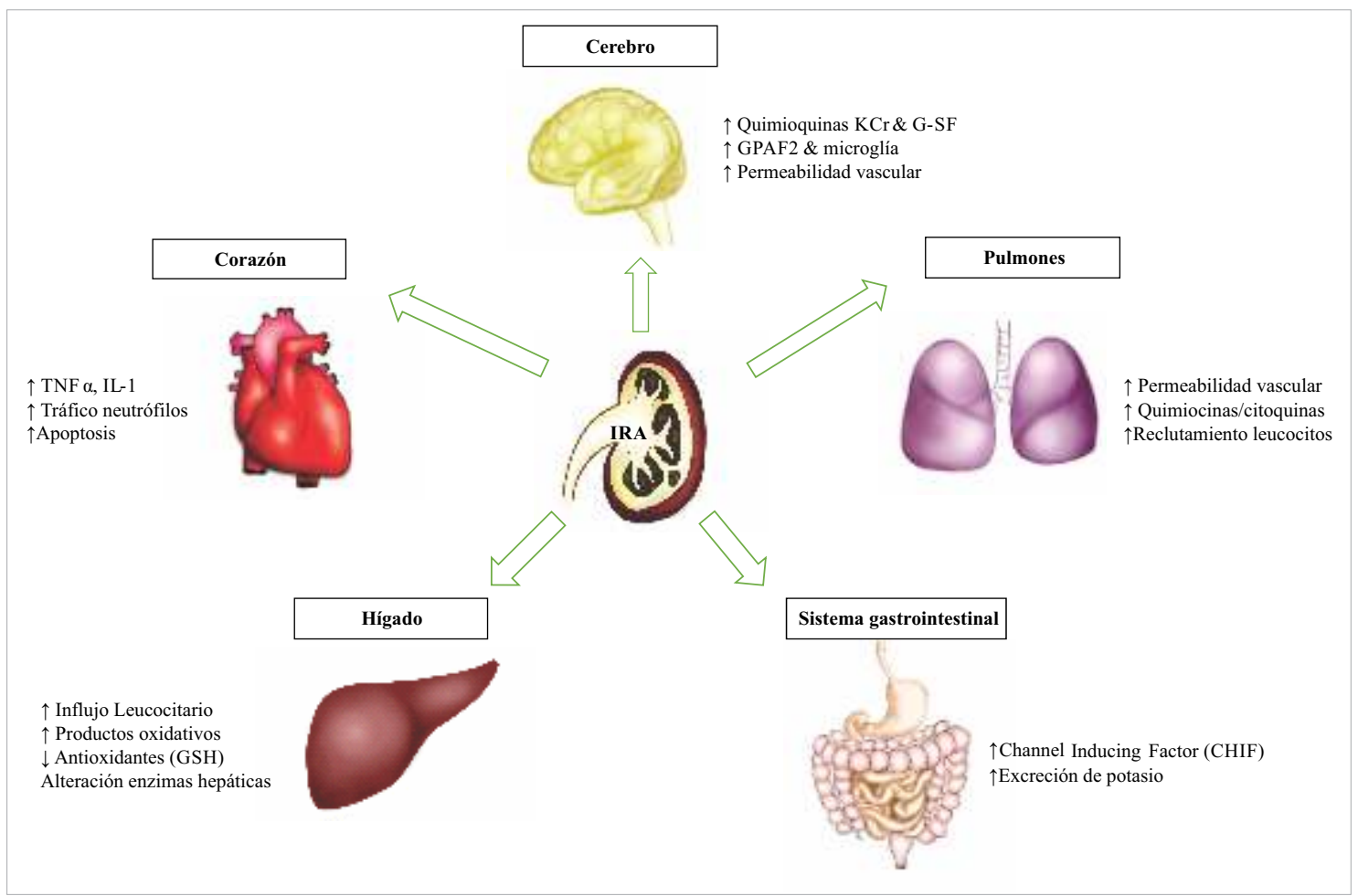

*TNF: Factor de necrosis tumoral, IL: Interleucina, CHIF: Factor inductor de canales iónicos, GSH: Glutation mitocondrial. Fuente: Elaboración de los autores.

Figura 3. Implicaciones extrarrenales de la falla renal aguda

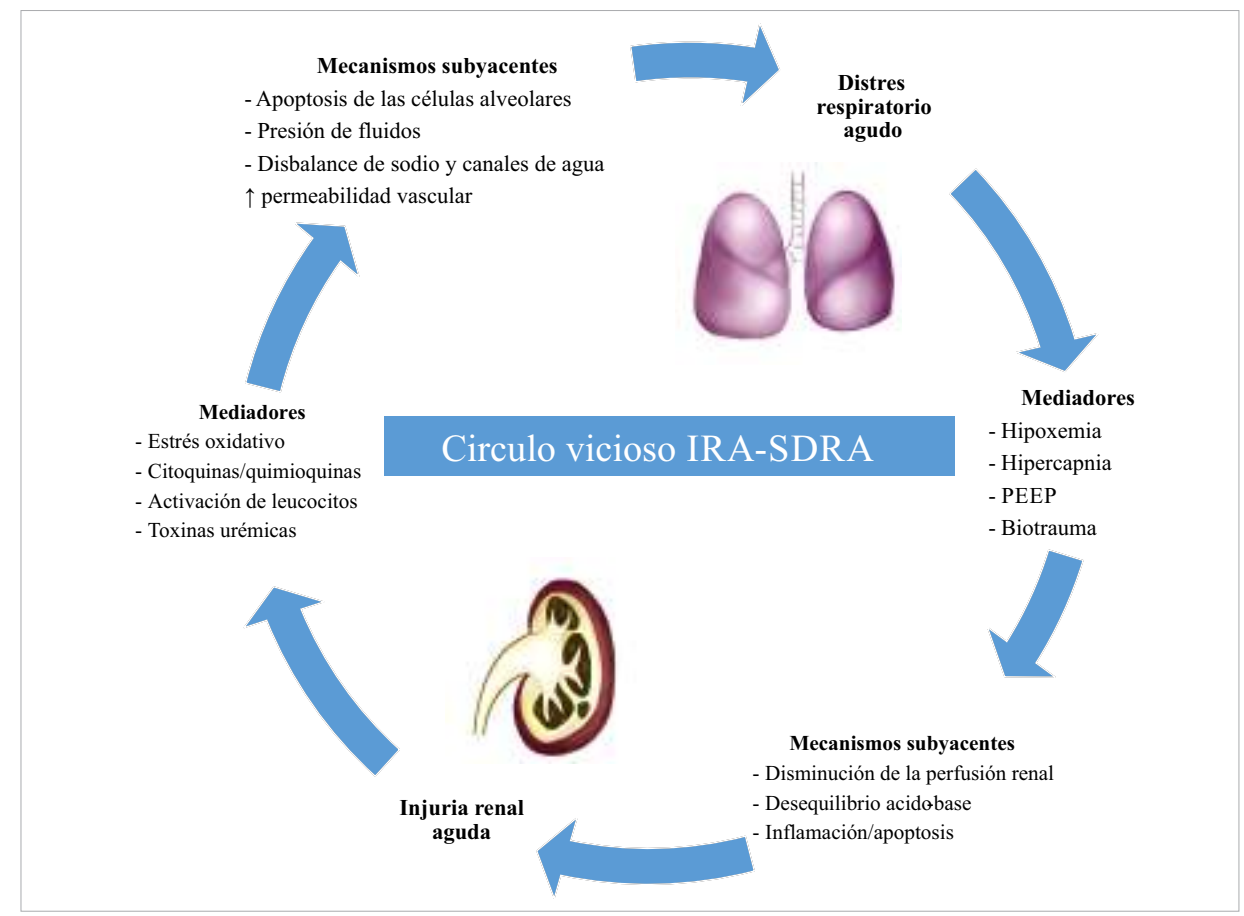

*PEEP: Presión positiva al final de la espiración.

Fuente: Elaboración de los autores.

Figura 4. Ciclo vicioso de la insuficiencia renal aguda y el síndrome de dificultad respiratoria del adulto. 
derivados artemisinina (artesunato y arteméter) y los alcaloides de la quina (quinina y quinidina) (28).

El estudio PALUREA, 154 pacientes con malaria grave, fueron tratados con quinina endovenosa, con una mortalidad del 5.2\% (29) (Tabla 3). La mortalidad se relaciona a múltiples factores, este paciente cursó con un binomio SDRA y falla renal asociado a sepsis de foco pulmonar (Figura 5). La relación entre la acidosis y SDRA es directa y eleva proporcionalmente la tasa de mortalidad (11). En una cohorte de 400 adultos con malaria severa importada, la mortalidad en la UCI fue del $10.5 \%$, tres variables presentes en la admisión en la UCI predijeron independientemente la muerte: edad avanzada, coma y mayor parasitemia (30).

\section{Conclusiones}

Es un caso de malaria importada, donde el paciente cursó con indicadores de mal pronóstico por malaria por $P$. falciparum. El compromiso multisistémico con Scores de gravedad muy elevados y progresión rápida de la necrosis tubular renal relacionada con el SDRA, así como lo describe la literatura, en insuficiencia renal aguda su intervención temprana se relaciona con mejoría del pronóstico, como también la terapia antimalárica establecida. La depuración temprana de la parasitemia, no descarta una eliminación total del parásito ya que puede haber presencia de este a nivel tisular debido a su secuestro esplénico, a pesar de tener manejo endovenoso dirigido.

Tabla 3. Primera línea de tratamiento de Malaria por P. Falciparum

\begin{tabular}{cc}
\hline Medicamento y presentación & Dosis y vía de administración en adultos \\
Artesunato IV & $\begin{array}{c}2.4 \mathrm{mg} / \mathrm{kg} \text { IV al ingreso (tiempo 0) y luego a las 12 y } \\
24 \text { horas. Continuar una vez al día hasta tolerar VO } 0 \\
\text { hasta completar } 7 \text { días }\end{array}$ \\
$\begin{array}{c}\text { Artemether + lumefantrine Tabletas de } \mathbf{2 0} \mathrm{mg} \text { de } \\
\text { artemether y } \mathbf{1 2 0} \mathrm{mg} \text { de Lumefantrine }\end{array}$ & $\begin{array}{c}\text { Al tolerar la vía oral: administrar } 6 \text { dosis en total, } \\
\text { distribuidas en dos tomas al día por tres días }\end{array}$ \\
\hline
\end{tabular}

Fuente: Elaboración de los autores

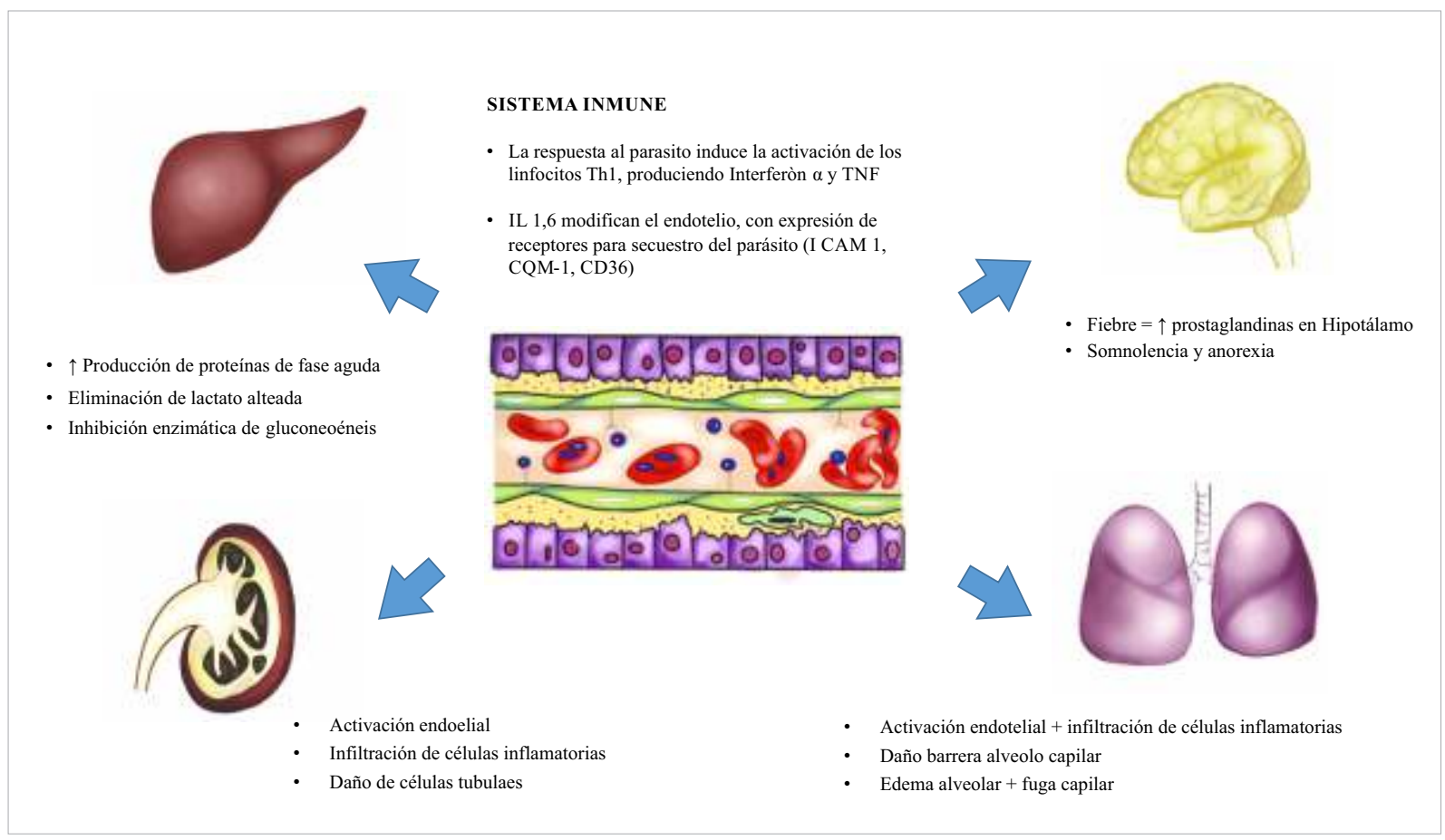

*Th1: Linfocito T Helper 1, TNF: Factor de necrosis tumoral, IL 1-6: Interleucina tipo 1 y tipo 6, I CAM: Moléculas de adhesión intracelular tipo 1, CQM-1: CD36: Linfocito Citotóxico 36.

Fuente: Elaboración de los autores.

Figura 5. Cascada inflamatoria por órgano blanco. 
El paciente fallece en el contexto de una falla multiorgánica, relacionando la actividad hiperinmune de los antígenos propios del parásito, los cuales generaban un fenómeno inflamatorio desenfrenado a los órganos implicados. Finalmente, el diagnóstico temprano y la terapia de soporte y dirigida de manera oportuna en estos pacientes, según la literatura muestra resultados alentadores respecto a mortalidad.

\section{Responsabilidades éticas}

Protección de personas y animales: Los autores declaran que para esta investigación no se han realizado experimentos en seres humanos ni en animales.

Confidencialidad de los datos: Los autores declaran que han seguido los protocolos de su centro de trabajo sobre la publicación de datos de pacientes.

Derecho a la privacidad y consentimiento informado: Los autores han obtenido el consentimiento informado del paciente referido en el artículo. Este documento obra en poder del autor de correspondencia.

\section{Conflicto de intereses}

Los autores declaran no tener conflicto de interés.

\section{Referencias}

1. Velázquez A, Álvarez F, Zavala C. Paludismo: Reporte de un caso y revisión de la literatura. Médica Sur, México. 2006;13(3): 129-135.

2. Nadjm B, Behrens R. Malaria: An Update for Physicians. Infectious Disease Clinics. 2012; 26(2): 243-259.

3. Fazil A, Vernekar P, Geriana D, Pant S, Senthilkumaran $S$, et al. Clinical profile and complication of malaria hepatopathy. Journal of Infection and Public Health. 2013; 6(5): 383-388.

4. Miranda A, Martínez E, Tobón A. El riñón en la malaria: de la patogénesis a las manifestaciones clínicas. Infectio. 2014; 18(3): 120-128.

5. World Health Organization. World malaria report 2012. [Internet] Geneva, 2012. [Cited: 4 July 2017]. Disponible en: http://www.who.int/malaria/publications/world_ malaria report 2012/en/

6. Instituto Nacional de Salud. Guía de Atención Clínica de Malaria. [Internet]. 2010. [Cited: 4 July 2017]. Disponible en: http://www.ins.gov.co/?idcategoria $=5620$

7. Ehrich J, Eke F. Malaria-induced renal damage: facts and myths. Pediatr Nephrol. 2007; 22(5): 626-637.

8. Blair S, Giraldo C. Complicaciones renales en la malaria. Acta Méd colombi. 2004; 29: 328-36.

9. Nguansangiam S, Day N, Hien T, Hoang N, Chaisri U et al. A quantitative ultrastructural study of renal pathology in fatal Plasmodium falciparum malaria. Tropical
Medicine \& International Health. 2007; 12(9): 10371050.

10. Chellappan A, Bhadauria D. Acute kidney injury in malaria: An update. Clinical Queries: Nephrology. 2016; 5(1): 26-32.

11. Bhadade R, Harde M, de Souza R, Khot A. Clinical characteristics and outcomes of patients with acute lung injury and ARDS. Journal of Postgraduate Medicine. 2011; 57(4): 286-290.

12. Van den Steen P, Deroost K, Deckers J, Van Herck E, Struyf S, Opdenakker G. Pathogenesis of malariaassociated acute respiratory distress syndrome. Trends in Parasitology. 2013; 29(7): 346-358.

13. Matthay M, Zemans R. The Acute Respiratory Distress Syndrome: Pathogenesis and Treatment. Annu Rev Pathol. 2011; 6(1): 147-163.

14. Tan L, Yacoub S, Scott S, Bhagani S, Jacobs M. Acute lung injury and other serious complications of Plasmodium vivax malaria. The Lancet Infectious Diseases. 2008; 8(7): 449-454.

15. Cheng M, Yansouni C. Management of Severe Malaria in the Intensive Care Unit. Critical Care Clinics. 2013; 29(4): 865-885.

16. Hanson J, Hasan M, Royakkers A, Alam S, Charunwatthana P, Maude R, et al. Laboratory prediction of the requirement for renal replacement in acute falciparum malaria. Malar J. 2011; 10:217. Disponible en: https://doi.org/10.1186/1475-2875-10-217

17. Marks M, Armstrong M, Suvari M, Batson S, Whitty C, Chiodini $\mathrm{P}$, et al. Severe imported falciparum malaria among adults requiring intensive care: a retrospective study at the hospital for tropical diseases, London. BMC Infect Dis. 2013; 13(118): 1-8.Disponible en: https:// doi.org/ 0.1186/1471-2334-13-118

18. Villa G, Ricci Z, Ronco C. Renal Replacement Therapy. Critical Care Clinics. 2015; 31(4): 839-848.

19. Instituto departamental de salud Norte de Santander. Análisis de situación de salud (ASIS) en municipios de frontera. [Internet]. 2010. [cited 4 July 2017]. pág. 96. Disponible en: https://www.minsalud.gov.co/ plandecenal/mapa/Analisis-de-Situacion-de-Salud-enmunicipios-de-frontera-Departamento-NorteSantander.pdf

20. Proyecto Fondo Mundial Malaria. Uso de la inteligencia epidemiológica con participación social, para fortalecer la gestión del programa, mejorar el acceso al diagnóstico y tratamiento y ejecutar intervenciones eficaces para la prevención y control de la malaria, Colombia 2010-2015. [Internet]. [Cited 4 july 2017]. Disponible en: http://simposiovirologia.ins.gov.co/ temas-de-interes/Documentacin \% 20Malaria/ Proyecto\%20Malaria\%20Colombia.pdf

21. Sagaki P, Thanachartwet V, Desakorn V, Sahassananda D, Chamnanchanunt S, et al. Clinical Factors for Severity of Plasmodium falciparum Malaria in Hospitalized Adults in Thailand. PLoS ONE [Internet]. 2013 [cited 4 July 2017]. 8(8):e71503. Disponible en: https://www.ncbi.nlm.nih.gov/pmc/articles/PMC374118 4/pdf/pone.0071503.pdf

22. Kute V, Shah P, Munjappa B, Gumber M, Patel H, Jain S, et al. Outcome and prognostic factors of malariaassociated acute kidney injury requiring hemodialysis: $\mathrm{A}$ 
single center experience. Indian J Nephrol. 2012; 22(1): 33-38.

23. Marks M, Gupta-WrightA, Doherty J, Singer M, Walker D. Managing malaria in the intensive care unit. British Journal of Anaesthesia [Internet]. 2014 [cited 4 July 2017]. 113(6):910-921. Disponible en: https://www. ncbi.nlm.nih.gov/pmc/articles/PMC4235570/pdf/aeu15 7.pdf

24. Saravu K, Rishikesh K, Kamath A. Determinants of mortality, intensive care requirement and prolonged hospitalization in malaria - a tertiary care hospital based cohort study from South-Western India. Malaria Journal [Internet]. 2014 [cited 4 July 2017]. 13(1):370. Disponible en: https://www.ncbi.nlm.nih.gov/pmc/articles/ PMC4177244/

25. Sahu S, Mohanty N, Rath J, Patnaik S. Spectrum of malaria complications in an intensive care unit. Singapore Medicine jorunal [Internet]. 2010 [cited 4 July 2017]. 51(3): 226-229. Disponible en: http://smj.sma.org.sg/5103/5103a5.pdf

26. Bruneel F, Tubach F, Mira J, Houze S, Gibot S, et al. Imported falciparum malaria in adults: host- and parasite-related factors associated with severity. The French prospective multicenter PALUREA cohort study. Intensive Care Medicine [Internet]. 2016 [cited 4 July 2017]. 42(10):1588-1596. Disponible en: https://www.ncbi.nlm.nih.gov/ pubmed/27169586.

27. Doctor S, Liu Y, Whitesell A, Thwai K, Taylor S, Janko M, et al. Malaria surveillance in the Democratic Republic of the Congo: comparison of microscopy, PCR, and rapid diagnostic test. Diagn Microbiol Infect Dis. 2016; 85(1): 16-18. Disponible en: http://dx.doi.org/10.1016/ j.diagmicrobio.2016.01.004.

28. World Healt Organization. Deungue: guidelines for diagnostic, treatment, prevention and control. [Internet]. 2009. [cited 4 July 2017]. Disponible en: http://www. who.int/tdr/publications/documents/denguediagnosis.pdf

29. Cheng M, Yansouni C. Management of Severe Malaria in the Intensive Care Unit. Critical Care Clinics [Internet]. 2013 [cited 4 July 2017]. 29(4):865-885. Disponible en: https://www.ncbi.nlm.nih.gov/pubmed/24094382

30. Bruneel F. Severe Imported Falciparum Malaria: A Cohort Study in 400 Critically III Adults. PLoS ONE [Internet]. 2010 [cited 4 July 2017]. 5(10):e13236. Disponible en: https://www.ncbi.nlm.nih.gov/pmc/articles/PMC295191 3/pdf/pone.0013236.pdf 